\title{
Stellar Prominences
}

\author{
A. Collier Cameron \\ School of Physics and Astronomy, University of St Andrews, North \\ Haugh, St Andrews, Fife, SCOTLAND KY16 9SS
}

\begin{abstract}
I review recent observational and theoretical progress in the study of condensations of cool material trapped in the coronae of rapidly rotating stars. Such condensations were discovered over a decade ago, producing transient moving absorption features in the optical Balmerseries lines. These condensations appear to be in a physical state similar to that of solar prominence material, in the sense that they are sufficiently cool and dense to contain substantial amounts of neutral hydrogen. The strong coronal magnetic fields of the stars in which they occur serve both to confine and to insulate the cool material. The coronal condensations (sometimes dubbed "slingshot prominences") form at a variety of distances from the star, but are observed to favour formation sites near the Keplerian co-rotation radius. Simple support models involving purely dipolar fields can explain the existence of stable mechanical equilibria for sheet-like prominences in the equatorial plane. More complex field configurations show a wider variety of stable formation sites, both inside the co-rotation radius and out of the stellar equatorial plane.
\end{abstract}

\section{Introduction}

Over the last 15 years, discoveries of condensations of neutral material in the coronae of late-type stars other than the Sun have led to the recognition that stellar coronae, like the solar corona, are multi-phase media. These stellar coronal condensations have often been dubbed "prominences", on the grounds that they appear to consist of dense, relatively cool material, embedded in the hot, ambient medium of a stellar corona and giving rise to optically thick Balmer emission or (more usually) absorption. The implied presence of strong magnetic fields in the active stars where they are observed, suggests that the condensations are both confined and supported by the field, and insulated by relatively poor cross-field thermal conductivity.

In this review, I shall begin with a brief survey of the observational evidence for stellar phenomena that have qualitative resemblances to quiescent prominences on the Sun. Mostly, however, I wish to emphasize the utility of the longer-lived stellar coronal condensations as tracers of stellar coronal structure. The spatial distribution of the condensations themselves provides us with a valuable diagnostic of the complexity of the coronal field topology in active stars. In the rapidly rotating young stars where the "slingshot prominences" occur, they serve as tracers of both the radial extent and longitudinal structure 
of the closed-field corona. When observed repeatedly, their evolution from one rotation cycle to the next provides us with detailed insights into the rate of evolution of the global topology of the coronal magnetic field.

\section{Coronal condensations in binary stars}

The earliest observational evidence for prominence-like structures in stellar coronae came from pencil-beam studies of eclipsing binary systems where a hot, compact companion is viewed through the corona of a larger, late-type companion for part of the orbit. Schröder (1983) attributed post-eclipse dips in the UV continuum of the eclipsing $\mathrm{K}$ supergiant + main-sequence B-star system 32 Cygni, to Rayleigh scattering in the HI ground state, and inferred a neutral $\mathrm{H}$ column density of $10^{24} \mathrm{~cm}^{-2}$ for a cool prominence-like structure located well above the stellar limb.

The eclipsing K0V + white dwarf binary V471 Tau is very well suited to such pencil-beam studies, having an orbital period close to half a day. Jensen et al (1986) reported the presence of soft X-ray dips repeating on successive orbits, at phases where the white dwarf was seen through the $\mathrm{K}$ star's corona, and inferred a column density $N_{H} \sim 10^{20} \mathrm{~cm}^{-2}$. At about the same time, Guinan et al (1987) found transient line absorption in UV lines including SiII, SiIII, Sirv, CII, CIII and CIV. This indicated a clumpy distribution of material at a variety of temperatures along the changing of sight to the white dwarf as it passed behind the $\mathrm{K}$ star's corona.

There is also strong evidence of intra-system material in many RS CVn systems. In particular, anomalous $\mathrm{H} \alpha$ absorption was seen in eight of ten eclipsing systems surveyed by Hall \& Ramsey $(1992,1994)$. Similar condensations, but at a temperatures closer to $2 \times 10^{4} \mathrm{~K}$, have been invoked by Steeghs et al (1996), as an explanation of peculiar low-velocity emission lines located near the inner Lagrangian points of the dwarf novae IP Peg and SS Cyg in their outburst states.

\section{Coronal condensations in single stars}

The other major line of investigation into stellar coronal condensations has been the study of $\mathrm{H} \alpha$ absorption transients in ultra-rapidly rotating young mainsequence stars with rotation periods in the range from 0.4 to $1.0 \mathrm{~d}$. Collier Cameron \& Robinson (1989a,b; CR89a,b) used time-resolved spectroscopy to infer the presence of clouds of $\mathrm{H} \alpha$-absorbing material in enforced co-rotation with the marginally pre-main sequence K0 star AB Doradûs.

The spatial distribution of $\mathrm{H} \alpha$ absorbing clouds around $\mathrm{AB}$ Dor and similar stars is easily inferred from time-series spectra. A typical time-series $\mathrm{H} \alpha$ observation is shown in Fig. 1 together with a simultaneous time-series showing the average photospheric line profile. In both time-series, transient features are seen to drift across the line profile, initially appearing blueshifted by approximately $-v \sin i$ from the stellar centre-of-mass velocity, and drifting toward the red until they disappear at $+v \sin i$. The photospheric line profiles exhibit bright "bumps" produced by dark spots on the stellar surface. The rates at which these features drift across the profile are therefore typical of features located on the 

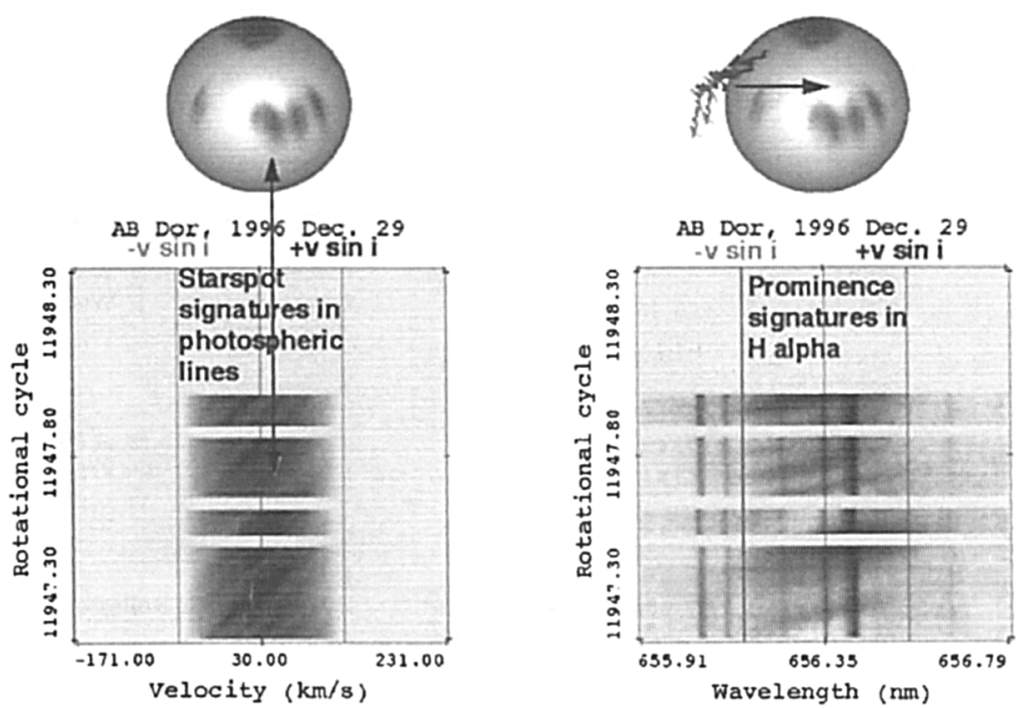

Figure 1. Spectral signatures of (a) photospheric starspots in combined profile of 1500 photospheric absorption lines, and (b) $\mathrm{H} \alpha$ absorption transients due to corotating circumstellar clouds, in time-series spectra secured at the AAT on 1996 December 29 by Donati et al (1999). The $\mathrm{H} \alpha$ transients cross the disc much faster than the stellar surface features.

stellar surface. The dark transient features seen in the $\mathrm{H} \alpha$ time-series cross the profile much faster, moving from $-v \sin i$ to $+v \sin i$ in little more than an hour in many cases. Since the features are seen to recur at intervals of one stellar rotation, the drift rate when the feature is in the centre of the profile is directly proportional to the distance of the feature from the stellar rotation axis:

$$
\dot{v}=\Omega^{2} \varpi \sin i
$$

where $\varpi$ is the cylindrical radial coordinate of the cloud, $\Omega$ is the star's rotational angular velocity, and $i$ is the inclination of the stellar rotation axis to the line of sight.

The drift rates of individual transients yield the distances of clouds from the rotation axis in units of the stellar equatorial radius, in terms of the directly measurable quantities $\dot{v}, v \sin i$ and $\Omega$ :

$$
\frac{\varpi}{R_{*}}=\frac{\dot{v}}{\Omega v \sin i}
$$

In their first studies of this phenomenon, CR89a,b found the clouds to lie at a range of distances from the stellar rotation axis, with a strong concentration near the co-rotation surface which in AB Dor lies some 2.7 stellar radii from the stellar rotation axis. Since then, the circumstellar cloud system of AB Dor has 
Table 1. Rapidly rotating (pre-) main-sequence stars surveyed to date, for $\mathrm{AB}$ Dor-like $\mathrm{H} \alpha$ absorption transients.

\begin{tabular}{|c|c|c|c|c|c|c|}
\hline Star & Sp.type & $P_{\text {rot }}$ & $v \sin i$ & $R \sin i$ & Proms? & References \\
\hline HE 373 & G3V & 0.33 & 140 & 0.92 & Yes & Collier Cameron \& Woods 92 \\
\hline HE 520 & G3V & 0.60 & 87 & 1.04 & Yes & $\begin{array}{l}\text { Collier Cameron } \& \text { Woods } 92 \\
\text { Barnes et al } 98\end{array}$ \\
\hline $\mathrm{HE} 622$ & G5V & 0.80 & 61 & 0.97 & Yes & Collier Cameron \& Woods 92 \\
\hline HE 699 & G1V & 0.33 & 90 & 0.59 & Yes & $\begin{array}{l}\text { Collier Cameron \& Woods } 92 \\
\text { Barnes et al } 98\end{array}$ \\
\hline AP 149 & G8V & 0.32 & 102 & 0.64 & Yes & Barnes 99 \\
\hline PZ Tel & $\mathrm{KOV}$ & 0.95 & 70 & 1.31 & Yes & Barnes et al 2000 \\
\hline AB Dor & $\mathrm{KOV}$ & 0.515 & 91 & 0.93 & Yes & $\begin{array}{l}\text { CR89a,b; } \\
\text { Collier Cameron et al 90; } \\
\text { Donati \& Cameron } 97 ; \\
\text { Donati et al } 99\end{array}$ \\
\hline Speedy Mic & $\mathrm{KOV}$ & 0.380 & 140 & 1.05 & Yes & Jeffries 93 \\
\hline $\mathrm{BD}+224409$ & K3V & 0.424 & 69 & 0.58 & No & $\begin{array}{l}\text { Jeffries et al } 94 \text {; } \\
\text { Eibe et al } 99\end{array}$ \\
\hline HK Aqr & M1V & 0.431 & 69 & 0.59 & Yes & Byrne, Eibe \& Rolleston 96 \\
\hline RE J1816+541 & M1V & 0.459 & 61 & 0.55 & Yes & Eibe 98 \\
\hline RX J1508.6-4423 & G2V & 0.31 & 115 & 0.70 & Yes & Donati et al 2000 \\
\hline
\end{tabular}

been observed almost annually, and similar phenomena have been observed in nearly a dozen stars by various authors whose efforts are summarized in Table 1 .

The presence of these condensations in a hotter and denser stellar corona than the Sun's suggests a formation mechanism similar to that of solar quiescent prominences. In a recent theoretical study of the thermal stability of stellar coronal loops, Ferreira \& Mendoza-Briceno (1997) found that the formation of fine, sheet-like condensations near the loop summits, with temperatures close to those observed, should be a common feature of such large loops. These occur for a wide variety of surface conditions; their precise locations relative to the loop summit were found to depend on whether or not the loop heating rate was symmetric about the summit.

While the fine dynamical and spatial structure of solar prominences is directly resolvable on Sun, only indirect measures are available on stars. The Balmer lines in most of the prominence-bearing dwarfs are optically thick, but with almost pure scattering source functions. By observing transients simultaneously in MgII h \& k, CaII H \& K and $\mathrm{H} \alpha$, Collier Cameron et at (1990) were able to establish that a typical prominence transient arises in a cloud with a column density $N_{H} \sim 10^{20} \mathrm{~cm}^{-2}$, at a temperature $T \sim 8000-9000 \mathrm{~K}$, and with a projected cross-sectional area $A \sim 3 \times 10^{21} \mathrm{~cm}^{2}$. This implies a total mass $M \sim 2-6 \times 10^{17} \mathrm{~g}$, two to three orders of magnitude greater than a typical solar quiescent prominence. There are typically 6 to 8 such condensations in the observable slice of the corona at any given time, at cylindrical radii ranging from 2 to $8 R_{*}$. New clouds form, and old ones disappear, on a timescale of 24 hours. Occasionally clouds are seen to move out to 6 to $8 R_{*}$ over the course of 1 to 2 days prior to their disappearance. This behaviour has been observed on three occasions, in 1986 November (CR89b), in 1994 November (Cameron et al 2000) and in 1996 December (Donati et al 1998).

Evidence for a wide variety of temperatures in the condensations emerged from recent HST observations (Brandt et al 2000) which showed transient ab- 
1998 Moy 06, original $\mathrm{H} \alpha$ dato

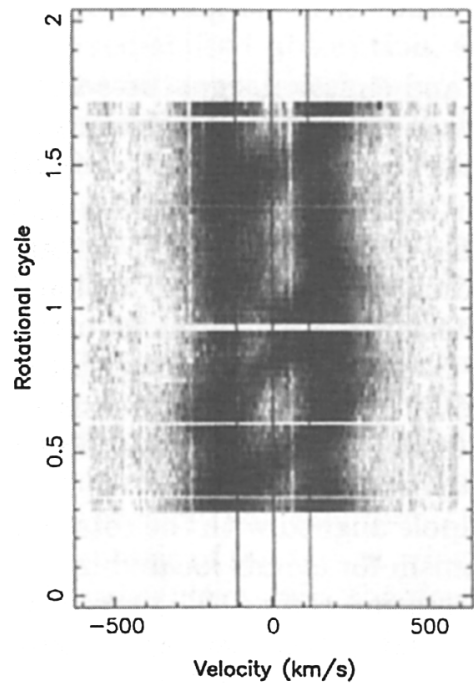

1998 May 06, Ha Doppler map

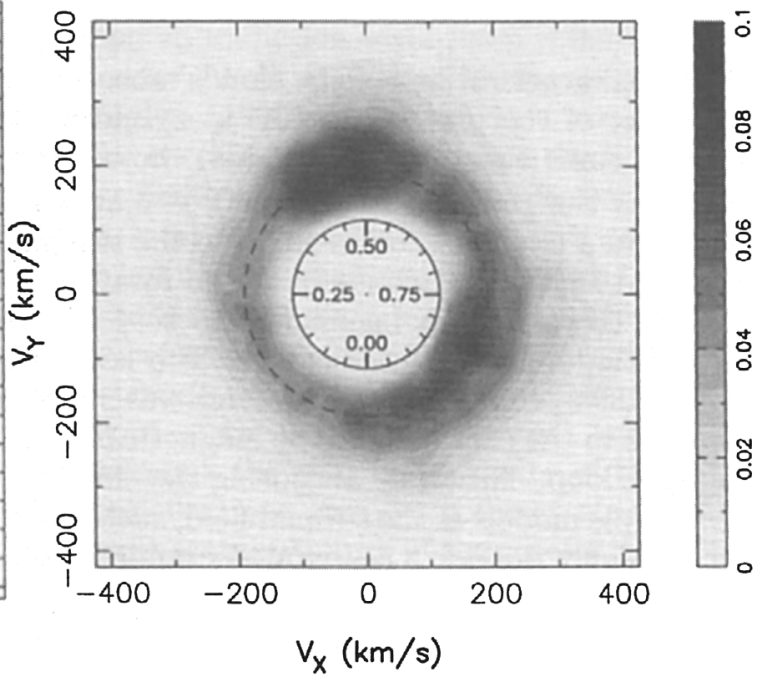

Figure 2. The trailed spectrogram of the $\mathrm{H} \alpha$ profile of RX J1508.6 -4423 , spanning 1.4 stellar rotations, shows clumps of emitting material tracing out sinusoidal paths around the star (Donati et al 2000). Tomographic back-projection (right panel) shows these clumps to be part of a ring of emiting material concentrated near the co-rotation radius (dashed circle).

sorption features in the UV resonance lines of CrV coinciding with $\mathrm{H} \alpha$ transients observed simultaneously from the ground. This observation is strongly reminiscent of the results obtained by Guinan et al in their pencil-beam study of V471 Tau, and it is tempting to conclude that both groups were observing similar coronal structures. Since the pressure scale height within such a prominence is expected to be of order hundreds of kilometres - three orders of magnitude less than the typical linear dimensions of the stellar condensations - a picture is beginning to emerge in which cool material at 8000 to $9000 \mathrm{~K}$ co-exists with material at both transition-region and coronal temperatures, possibly in filamentary sub-structures. This picture is once again broadly consistent with what we see in solar quiescent prominences.

In extreme rotators where the prominences form close to the stellar surface, however, the increased EUV and soft X-ray radiation field can drive the Balmer lines into emission. In this case, the prominence signatures produce a characteristic double-peaked emission profile. This phenomenon has been seen in both the pre-main sequence G2 star RX J1508.6 -4423 (Donati et al 2000) and the G8 dwarf AP 149 in the $\alpha$ Persei cluster (Barnes 1999). Tomographic back-projection shows that in both cases the emission originates in a clumpy ring of emitting material, corotating with the star and strongly concentrated near the co-rotation radius (Fig. 2). 


\section{Magnetic confinement}

The radial accelerations of the clouds' absorption and emission signatures show that most of the prominences lie at cylindrical radii $\varpi$ near (but some inside and and some substantially outside) the equatorial co-rotation radius, defined simply as the radius $a=\left(G M_{*} / \Omega^{2}\right)^{1 / 3}$ at which the orbital period of a test particle in a circular orbit is equal to the star's axial rotation period. If a cloud is located significantly outside the co-rotation radius, the inward gravitational force on the plasma is insufficient to keep its material in a synchronous orbit. An additional inward force is needed to keep the cloud in co-rotation with the star. CR89a proposed that this force was supplied by the the magnetic tension force due to the curvature of the magnetic field lines near the summit of a closed magnetic loop, effectively anchoring the cloud to the stellar surface.

Simple models of the coronal field, such as a dipole aligned with the rotation axis, can only provide a centrifugal support mechanism for clouds located in the stellar equatorial plane and outside the stellar co-rotation radius. The presence of clouds at radii substantially inside the co-rotation radius has been reported by Byrne, Eibe \& Rolleston (1996) in the single M1V rapid rotator HK Aqr, and by Eibe (1998) in the single M1V rapid rotator RE J1816+541. Moreover, many of the prominence-bearing dwarfs are viewed at inclination angles such that clouds confined to the equatorial plane outside the co-rotation radius would not cross the stellar disc. To explain why absorption transients are seen in such a wide range of moderately-inclined stars, we need to examine more complex magneticfield configurations.

Ferreira (2000) pointed out that in a stable equilibrium site capable of supporting a prominence, the component of effective gravity (incorporating centrifugal forces) along the direction of the local field must be zero such as to give a local gravitational-centrifugal potential minimum. He found that such stable equilibria can exist inside corotation for multipolar field configurations, and considerably above or below the stellar equatorial plane if the field has a strong quadrupolar component. A preliminary study by Jardine et al (2000) used potential-field extrapolation from the Zeeman-Doppler images of Donati et al (1999) to explore the complexity of the large-scale field. Their results show that stable prominence support sites satisfying the equilibrium condition $B . g_{\text {eff }}=0$ and the stability criterion $(B . \nabla)\left(B . g_{\text {eff }}\right)<0$ can be found well inside corotation and out of the equatorial plane. Here $g_{\text {eff }}$ includes centrifugal forces acting on the loop plasma in the co-rotating frame.

There do, however, appear to be limits to the distance above the equatorial plane at which prominence support is possible. The main evidence for this comes from the lack of observed prominence activity in the rapidly rotating mid-K dwarf $\mathrm{BD}+22^{\circ} 4409$, whose axial inclination is, at $45^{\circ}$ to $50^{\circ}$ (Jeffries et al 1994), demonstrably the lowest among the ultra-fast rotators in the solar neighbourhood. In a detailed study of $\mathrm{H} \alpha$ profiles secured over two nights in 1993 August, Eibe et al (1999) found no evidence for transient AB Dor-like absorption features. They found, however, that the $\mathrm{H} \alpha$ emission profile is anomalously narrow, with a full width at half maximum intensity substantially less than the stellar $v \sin i$. They also remarked that the profile was asymmetric to the blue at all rotation phases, suggesting that the chromospheric emission profile 
is partially masked by persistent red-shifted absorption arising in a curtain of down-flowing material containing significant amounts of neutral hydrogen. Similar red-shifted absorption, suggesting sporadic low-level mass infall, has been noted in several weak-line T Tauri stars (Wolk \& Walter 1996), and in II Peg (Byrne 1987). This led Walter \& Byrne (1998) to suggest that cool material may be continually condensing in the corona. This idea makes the implicit assumption that local density enhancements high in the corona suffer increased radiative losses which can trigger a local thermal collapse. The growing cool condensation is subsequently fed by hot coronal material blowing up the legs of the now under-pressured loop. The prominence mass is thus supplied from the stellar surface via upflows, the Coriolis forces on which may even destabilize the loop unless the field is sufficiently strong (Ferreira \& Jardine 1996). If indeed material is continually condensing out of the large-scale corona, and settling temporarily in whatever potential mimima the shifting geometry of the corona can provide, the evolution of the condensations provides a direct observational diagnostic of the rate at which the large-scale structure of the corona is evolving. Elsewhere, however, the dense unsupported material will rain back down on to the stellar surface, giving red-shifted absorptions at velocities approaching the free-fall value.

\section{Summary and future prospects}

Coronal condensations show great promise as a tool for probing the radial extent and geometric complexity of the closed-field coronae surrounding rapidly rotating late-type stars. The fact that prominences are observed both within the co-rotation radius, and in regions located significantly above and below the equatorial plane, requires complex field topologies to provide mechanical support support. The red-shifted Balmer-line absorption seen in $\mathrm{BD}+22^{\circ} 4409$ and many RS CVn systems suggest that coronal condensations form throughout the extended stellar corona in these systems too, giving continuous downflows at high latitudes and in other regions where the field cannot give mechanical support. The global structure of the prominence system appears to evolve faster than surface structure. Whether this indicates that the coronal field is continually destabilised by surface shear, or whether other mechanisms are needed to drive the evolution of the corona, remains an open question. Nonetheless, the combination of global field models derived from Zeeman-Doppler imaging with prominence evolution studies is proving to be a powerful method for studying the evolving three-dimensional topology of active stellar coronae.

\section{References}

Barnes J. R., Collier Cameron A., Unruh Y. C., Donati J.-F., Hussain G. A. J., 1998, MNRAS, 299, 904

Barnes J. R., 1999, PhD thesis, University of St Andrews

Barnes J. R., Collier Cameron A., James D. J., Donati J.-F., 2000, MNRAS, 314,162

Brandt J. C., et al., 2000, AJ, submitted 
Byrne P., Eibe M., Rolleston W., 1996, A\&A, 311, 651

Byrne P. B., 1987, in Linsky J. L., Stencel R. E., eds, Lecture Notes in Physics, Vol. 291: Cool Stars, Stellar Systems and the Sun. Springer-Verlag, Berlin, p. 491

Collier Cameron A., Robinson R. D., 1989a, MNRAS, 236, 57 (CR89a)

Collier Cameron A., Robinson R. D., 1989b, MNRAS, 238, 657 (CR89b)

Collier Cameron A., Woods J. A., 1992, MNRAS, 258, 360

Collier Cameron A., Duncan D. K., Ehrenfreund P., Foing B. H., Kuntz K. D., Penston M. V., Robinson R. D., Soderblom D. R., 1990, MNRAS, 247, 415

Collier Cameron A., et al., 1999, MNRAS, 308, 493

Donati J.-F., Collier Cameron A., 1997, MNRAS, 291, 1

Donati J.-F., Collier Cameron A., Hussain G. A. J., Semel M., 1999, MNRAS, 302,437

Donati J.-F., Mengel M., Carter B. D., Marsden S., Collier Cameron A., Wichmann R., 2000, MNRAS, 316, 699

Eibe M. T., Byrne P. B., Jeffries R. D., Gunn A. G., 1999, A\&A, 341, 527

Eibe M. T., 1998, A\&A, 337, 757

Ferreira J. M., Jardine M., 1996, A\&A, 305, 265

Ferreira J. M., Mendoza-Briceno C., 1997, A\&A, 327, 252

Ferreira J. M., 2000, MNRAS, 316, 647

Guinan E. F., Wacker S. W., Baliunas S. L., Loeser J. G., Raymond J. C., 1987, in New Insights in Astrophysics: Eight Years of UV Astronomy with IUE. ESA SP-263, p. 197

Hall J. C., Ramsey L., 1992, AJ, 104, 1942

Hall J. C., Ramsey L., 1994, AJ, 107, 1149

Jardine M., Collier Cameron A., Donati J.-F., Pointer G. R., 2000, MNRAS, submitted

Jeffries R. D., Byrne P. B., Doyle J. G., Anders G. J., James D. J., Lanzafame A. C., 1994, MNRAS, 270, 153

Jeffries R. D., 1993, MNRAS, 262, 369

Jensen K. A., Swank J. H., Petre R., Guinan E. F., Sion E. M., Shipman H. L., 1986, ApJ, 309, L27

Schröder K. P., 1983, A\&A, 124, L16

Steeghs D., Horne K., Marsh T. R., Donati J. F., 1996, MNRAS, 281, 626

Unruh Y., Jardine M., 1997, A\&A, 321, 177

Walter F. M., Byrne P. B., 1998, in Tenth Cambridge Workshop on Cool Stars, Stellar Systems, and the Sun. ASP Conference Series, San Francisco, p. 1458

Wolk S. J., Walter F. M., 1996, AJ, 111, 2066 